\title{
Medicine goes gender: eine Provokation
}

\section{Kathrin Zehnder}

Korrespondenz: Kathrin Zehnder HSA Luzern

Postfach 5232 Werftestrasse 1 CH-6002 Luzern

kzehnder@hsa.fhz.ch
Im beschriebenen Fall werden zwei Lösungsmöglichkeiten erläutert, die einschneidende operative Eingriffe mit sich bringen. Einem Kind sollen gesunde Ovarien und ein funktionsfähiger Uterus entfernt bzw. ein Penis zu einer Klitoris «reduziert» werden. Die Geschlechtsoperationen stellen keine Notfallmassnahmen dar, sind also aufschiebbar, jedoch nicht rückgängig zu machen. Es scheint auf den ersten Blick unerklärlich, warum überhaupt darüber nachgedacht wird, solche Eingriffe bei einem Fünfjährigen vorzunehmen. Im hier geschilderten Fall wird nämlich, so könnte man behaupten, weniger aus dem medizinischen Paradigma als vielmehr aus einem gesellschaftlichen Dilemma heraus argumentiert.

Unsere Geschlechterordnung geht in ihren Grundzügen von zwei Prämissen aus. Erstens: Geschlecht besteht in genau zwei Ausprägungen, der männlichen und der weiblichen. Zweitens: Das Geschlecht ist unveränderbar. Die zweite Grundlage hat sich in den Jahren nach «Agnes»a etwas aufgeweicht. Heute ist es möglich, unter ganz bestimmten Bedingungen sein Geschlecht zu verändern. Die erste Prämisse jedoch blieb bisher beinahe unumstritten. ${ }^{\mathrm{b}}$

Trans- und Intersexualität unterscheiden sich fundamental. Während die Transsexualität als rein psychische Erscheinung der Geschlechtsidentität definiert wird, bezeichnet die Medizin die Intersexualität als somatisches Phänomen. Beide Erscheinungen haben indessen eine Gemeinsamkeit: Sie werden auf der Grundlage der beschriebenen Prämissen als Krankheitsbilder erfasst. Anders gesagt: Eine bestimmte Kombination von in Erscheinung tretenden Elementen sind nicht per se Krankheiten, sondern werden von der diesbezüglich fachkundigen Gemeinschaft von Medizinerinnen und Medizinern als Trans- oder Intersexualität definiert. ${ }^{c}$ Genauso wie die Androgenisierung des kleinen Jungen im beschriebenen Fall natürlich kein «Teufelskreis» ist, sondern erst durch die Festlegung dessen, was erwünscht ist und was vermieden werden soll, als solcher bezeichnet wird, ist die Auslegung eines uneindeutigen Genitales als Störung nur eine mögliche Interpretation, die aufgrund unserer kulturellen Realität sinnhaft erscheint. ${ }^{\mathrm{d}}$ Aus der zweiten Prämisse («es gibt nur männlich oder weiblich») und der damit verbundenen Definition der Intersexualität als Störung oder Krank- heit folgt, dass ein Mensch mit äusserem männlichem Genital keine inneren weiblichen Genitalanlagen haben kann bzw. dass diese als «störend» interpretiert werden müssen (erste Lösungsmöglichkeit). Aufgrund der ersten Prämisse wiederum («das Geschlecht ist grundsätzlich unveränderbar») kann im Alter von sechs Jahren ein Geschlechtswechsel nicht vollzogen werden, zumal die definierte Ausnahme der Transsexualität nicht vorliegt. Die Autorinnen und Autoren der Fallgeschichte beschreiben folglich aus dieser Optik die Irritation einer gesellschaftlichen Regel und nicht in erster Linie eine medizinische Fragestellung. Aus der Irritation der Regel wird eine Bestätigung der Regel, indem, durch das uneindeutige Genitale, korrigiert bzw. eine Angleichung von äusseren und inneren Geschlechtsmerkmalen vorgenommen wird. Damit wird «Normalität» (wieder-)hergestellt und die zweite Prämisse bestätigt: Es gibt nur zwei Geschlechter.

Im vorliegenden AGS-Fall scheint sofort klar, dass damit, dass das Kind «jetzt und später möglichst wohl in seiner Geschlechtsrolle sicher und zufrieden aufwachsen kann», nur eine Geschlechtsrolle als Mann oder Frau gemeint sein kann. Eine intersexuelle Geschlechtsrolle wird (im Gegensatz zur körperlichen Intersexualität) nicht nur als Störung bezeichnet, sondern ist nicht existent. Würde man intersexuell geborene Kinder nicht operativ an ein Geschlecht angleichen, würde man im Alltag auch vermehrt solchen «Mischwesen» begegnen. Die Folgen davon könnten eine Aufweichung unserer Geschlechterordnung bedeuten. Damit könnte auch eine Geschlechterrolle fern einer männlichen oder weiblichen denkbar werden. Würde man damit der Besonderheit intersexueller Kinder nicht viel mehr gerecht?

Diesen Überlegungen könnte man entgegenhalten, dass es ein Experiment wäre, ein Kind mit zwittrigem Genitale aufwachsen zu lassen, weil die Zusammenhänge zwischen dem geschlechtlichen Phänotyp und der psychischen Geschlechtsentwicklung nur sehr wenig erforscht sind. Wie sich die Geschlechtsidentität eines intersexuellen Menschen entwickelt (bzw. entwickeln würde, wäre er nicht kurz nach seiner Geburt operativ an das ihm zugeteilte Geschlecht angepasst worden), ist ebenfalls unklar. Solange dies so ist, scheint es doch vielmehr 
experimentellen Charakter zu haben, ein Kind körperlich an ein Geschlecht anzugleichen, in der Annahme, es könne nur so eine gesunde Geschlechtsidentität entwickeln. Bräuchte es nach dem Prinzip des Nichtschadens nicht eine stichhaltige Begründung für einen Eingriff statt gegen ihn? Wenn auch nur leiser Zweifel an der Richtigkeit der Operationen besteht, müssen diese nicht konsequenterweise unterlassen werden? Wäre es denn nicht denkbar, dass ein Mensch «wohl in seiner Geschlechterrolle» sein könnte, auch wenn er diese neu definieren muss? Wäre es nicht ebenfalls denkbar, dass auch ein Mensch mit Geschlechtsmerkmalen beider Geschlechter eine eindeutig weibliche oder männliche Geschlechtsidentität entwickelt? Hinweise auf diese Fragen können intersexuelle Erwachsene geben. Sie müssen als Expertinnen und Experten in den Diskussionen herangezogen und als kundig bezüglich ihrer Besonderheit anerkannt werden. Viele Betroffene äussern sich heute kritisch bezüglich der an ihnen vorgenommenen Operationen. Diese Äusserungen werden noch viel zuwenig berücksichtigt und/oder systematisch untersucht.

Ein weiterer Mangel in der Fallbeurteilung scheint der wenig differenzierte Einbezug von Sexualität und sexueller Orientierung bzw. das Vermischen verschiedener Aspekte von Geschlecht ${ }^{\mathrm{f}} \mathrm{zu}$ sein: In den beschriebenen Lösungsmöglichkeiten wird von der sexuellen Funktion des künftigen Mannes gesagt, sie sei «unsicher, wahrscheinlich aber möglich». Sexuelle Funktion, so lässt sich vermuten, meint die Möglichkeit, heterosexuellen Geschlechtsverkehr zu haben, also Penetrationsfähigkeit. Bei der zweiten Lösungsvariante hingegen wird die - in diesem Falle weibliche - sexuelle Funktion nicht erwähnt. Diese kann aber bei einer Klitorisreduktion ebenfalls nicht garantiert werden. ${ }^{g}$ Wenn Sexualität als Lustempfinden und evtl. Orgasmusfähigkeit verstanden wird (und nicht lediglich als heterosexuelle Penetration der weiblichen Vagina durch einen männlichen Penis), hat die Vagina zudem weniger sexuelle als vielmehr Fortpflanzungsfunktion, während der Penis als Organ beide Funktionen erfüllt. Schliesslich ist eine homosexuelle Orientierung denkbar. Weshalb die Autorinnen und Autoren eine solche nur bei einem Aufwachsen als Frau bedenken, ist unverständlich, und warum eine solche in die Reihe mit fehlenden oder unzufriedenen geschlechtlichen Beziehungen eingereiht wird, ist mehr als fraglich, wenn nicht gar homophob. ${ }^{\mathrm{h}}$

Zusammenfassend lässt sich sagen, dass:

a. einmal durchgeführte Operationen nicht rückgängig gemacht werden können, gesunde
Organe mit einer Operation entfernt oder verstümmelt werden;

b. wir so gut wie nichts wissen über ein intersexuelles Bewusstsein oder die geschlechtliche Entwicklung von Menschen mit intersexuellen Organen. Solange es keine klinischen Studien gibt, müssen wir uns auf die Erfahrungsberichte von betroffenen Erwachsenen stützen, zumindest müssen diese durchaus kontroversen Stimmen - mitberücksichtigt werden; ${ }^{i}$

c. sexuelle Funktionsfähigkeit weit mehr ist als heterosexuelle Penetration;

d. ein konstruiertes Geschlechtsorgan bezüglich seiner sexuellen «Nutzbarkeit» niemals wie ein natürliches «funktioniert»;

e. Kinder, die nicht operiert werden, später die Möglichkeit haben, selbst zu entscheiden, was sie sein wollen;

f. verschiedene Aspekte von Geschlecht nicht unreflektiert vermischt werden dürfen sowie Stigmatisierungen bezüglich weiblicher und homosexueller Sexualität aufgedeckt und verbalisiert werden müssen. Und schliesslich, dass

g. gesellschaftliche Fragen nicht mit medizinischen Massnahmen beantwortet werden dürfen.

Natürlich ziehen diese Überlegungen in ihrer Konsequenz keinen einfachen Weg nach sich. Intersexuelle Kinder werden vielleicht gehänselt, lässt man sie in ihrer Uneindeutigkeit (obwohl auch dazu keine Untersuchungen existieren und Hänseleien aufgrund anderer Merkmale nicht ausgeschlossen werden können). Nichtoperierte intersexuelle Menschen werden sich mühsam einen Platz in unserer Geschlechterordnung erkämpfen müssen. Die Aufklärung und Begleitung der Kinder wäre mit grossem Aufwand verbunden, und intersexuelle Kinder bedürften eines besonderen Schutzes. Intersexuelle Kinder können nicht durch Operationen «normalisiert» werden, í sondern nur durch die Akzeptanz der Besonderheit bzw. die Enttabuisierung der Thematik. Eltern, Ärztinnen und Ärzte, Psychologinnen und Psychologen sowie Sozialarbeitende haben die Pflicht, das Kind bei diesem Prozess zu unterstützen. Statt der eindeutigen Zuteilung zu einem Geschlecht, wie in den beiden beschriebenen Lösungen, muss das Bestreben im vorliegenden Fall dahin gehen, den kleinen Jungen zu stärken und ihm und seiner Familie ${ }^{k}$ die optimale Unterstützung zu bieten. Das heisst nicht nur - wie in der «theoretischen Möglichkeit» beschrieben -, eine Entscheidung für die männliche Rolle zu fällen, sondern den Knaben über seine Besonderheit, sprich seinen weiblichen Anteil, 
altersgemäss aufzuklären und ihm so den Weg für eine eigene Entscheidung bezüglich seiner Geschlechtszugehörigkeit zu bahnen.

\section{Fussnoten}

a Die von Garfinkel [1] erstmals in der Literatur beschriebene Mann-zu-Frau-Transsexuelle.

b Sie wird durch die Existenz der Transsexualität gar gefestigt und bestätigt, denn Transsexualität ist, laut ICD-10, eine psychische Störung in Form eines dauerhaften Wunsches, ins gegenteilige Geschlecht $\mathrm{zu}$ wechseln. Veränderung ist also lediglich denkbar vom Mann zur Frau oder umgekehrt. Während die Medizin auf die erwähnten Irritationen der Geschlechterordnung mit der Klassifikation als diagnostizierbare Krankheit und einer entsprechenden Behandlung begegnete, womit die Dichotomie des Geschlechts nicht in Frage gestellt wurde, wird diese von den Geistes- und Gesellschaftswissenschaften zunehmend hinterfragt. Man versucht aufzuzeigen, dass die medizinische Definition von Transsexualität der Geschlechtsempfindung Transsexueller nur in beschränktem Masse gerecht wird, dass sie zu eng gefasst ist und dass es die Transsexualität eigentlich nicht gibt (vgl. z. B. Hirschauer [2]; Franzen und Berger [3]). Keinesfalls ist nämlich für transsexuelle Menschen immer klar, dass sie im gegenteiligen Geschlecht leben wollen. Häufig schliessen sie lediglich ein Leben in ihrem ursprünglichen Geschlecht als Mann oder Frau aus, leben aber eine Vielzahl verschiedener Geschlechterentwürfe und sind keinesfalls «eindeutig» Mann oder Frau.

c Im Falle des AGS entsprechen die einzelnen Elemente beispielsweise der beschriebenen Androgenproduktion oder der Virilisierung des Fetus. Im Vergleich zu einer durchschnittlichen Androgenproduktion wird diejenige im AGS-Fall als übermässig und somit die damit verbundene Virilisierung als krankhaft festgelegt. Mit AGS ist in vielen Fällen ein lebensgefährlicher Salzverlust verbunden was eine Klassifizierung als Störung des gesamten Komplexes von Erscheinungen noch selbstverständlicher zu machen scheint.

d Man kann sich durchaus vorstellen, dass ein Kind, das mit vier Jahren schon Schamhaare hat oder mit neun Jahren eine Brust entwickelt, in anderen Kulturkreisen als «Wunderkind» gesehen würde, genauso wie in unserem Kulturkreis ein Kind, das mit fünf Jahren den Satz des Pythagoras versteht (vgl. dazu etwa die Ausführungen von Herdt [4] zum sexuellen Dimorphismus in Kultur und Geschichte).

e Interessanterweise werden die beschriebenen geschlechtsangleichenden Operationen ja erst seit den 1950er Jahren vorgenommen. Darüber, wie es intersexuellen Menschen vor diesem Zeitpunkt im Vergleich zu den operativ behandelten unserer Zeit ging, ist genausowenig bekannt wie zur Frage, was die Medizin zu den Operationen (abgesehen vom Machbarkeitskriterium) veranlasst hatte. Als Wendepunkt sind evtl. die heute mehr als umstrittenen Untersuchungen von John Money aus den 1960er Jahren und seine Theorie der Geschlechtsneutralität im Kindesalter auszumachen. Allerdings gibt es dazu keine systematischen Untersuchungen. f Zur Abgrenzung von sexueller Identität, sexueller Präferenz und sexueller Orientierung sowie der Definition von Geschlechtsrolle, Geschlechtsidentität und Geschlechtsrollenidentität vgl. Richter-Appelt [5].

g Zudem bedingt das Anlegen einer Vagina das lebenslange Bougieren derselben. Die Folgen davon und die Auswirkungen der zahlreichen Nachbehandlungen von Geschlechtsoperationen können traumatisierend wirken und sind wenig untersucht.

h Abgesehen davon zeigt eine aktuelle Studie der Hamburger Forschergruppe keine solche Häufung von «gleichgeschlechtlichen» Partnerschaften bei AGS-PatientInnen, wenn auch die Stichprobe sehr klein war [5].

i Vgl. beispielsweise http://65694.rapidforum.com.

j Es ist zum Beispiel vollkommen unklar, ob ein Kind, das äusserlich einem eindeutigen Geschlecht angeglichen wurde, damit auch körperlich und psychisch als «normal» gelten würde. Vielleicht hätte es als Junge trotz Operation einen zu kleinen Penis, als Mädchen eine zu grosse Klitoris. Das Kind müsste sich ständig untersuchen und allenfalls nachbehandeln lassen. Es wäre eben doch nicht so wie alle anderen Kinder.

k Im beschriebenen Fall kommt als besondere Komponente die ethnische Zugehörigkeit des Patienten dazu. Die im Fall erwähnte Tatsache, dass auf dem Balkan sogenannte «sworn virgins» existieren, könnte eine Chance sein, dass auch ein Knabe mit teilweise weiblichen Geschlechtsmerkmalen gesellschaftlich anerkannt leben kann. «Sworn virgins» sind Frauen, die als Männer leben, männliche Kleidung tragen und Männerarbeit verrichten. «Sworn virgins» deshalb, weil sie sich zu ewiger Jungfräulichkeit verpflichten. Häufig werden «sworn virgins» in Familien ohne männliche Erben bereits von frühester Kindheit als Junge erzogen. Andere wählen nach einer weiblichen Sozialisation den Statuswechsel zum Mann selber, z.B., um sich einer arrangierten Heirat zu entziehen oder das Erbe anzutreten (vgl. Grémaux [6]). Jedoch gilt zu bedenken, dass die Rolle einer «sworn virgin» eine rigide Beschneidung der partnerschaftlichen Beziehungen mit sich bringt. Der im Fall beschriebene Junge dürfte nach dieser Tradition weder eine heteronoch eine homosexuelle Beziehung eingehen.

\section{Literatur}

1 Garfinkel H. Studies in ethnomethodoloy. Englewood Cliffs (NJ): Prentice Hall; 1967.

2 Hirschauer S. Die soziale Konstruktion der Transsexualität: über die Medizin und den Geschlechtswechsel. Frankfurt a. M.: Suhrkamp; 1993.

3 Franzen J, Beger NJ. «Zwischen die Stühle gefallen»: Ein Gespräch über queere Kritik und gelebte Geschlechterentwürfe. In: polymorph (Hrsg.). (K)ein Geschlecht oder viele?: Transgender in politischer Perspektive. Berlin: Querverlag; 2002. S. 53-68.

4 Herdt G (ed.). Third Sex Third Gender: Beyond Sexual Dimorphism in Culture and History. New York: Zone Books; 1994.

5 Richter-Appelt H. Intersexualität und Medizin. Zeitschrift für Sexualforschung. 2004;17:239-57.

6 Grémaux R. Woman Become Man in the Balkan. In: [4]. p. 241-81. 